\title{
Variable temperature effects on TEG performance
}

\author{
Björn Pfeiffelmann ${ }^{1}$, Cansu Özman ${ }^{1}$, Ali Cemal Benim ${ }^{1, *}$, and Franz Joos ${ }^{2}$ \\ ${ }^{1}$ Center of Flow Simulation (CFS), Dept. Mechanical and Process Engineering, Düsseldorf University of Applied Sciences, Germany \\ ${ }^{2}$ Laboratory of Turbomachinery, Helmut Schmidt University, Hamburg, Germany
}

\begin{abstract}
The present paper presents an experimental investigation of the variable temperature effects on the performance of a Thermoelectric Generator (TEG). In the conducted experiments, a sample TEG is analyzed by imposing variable temperature patterns on the cold side, while keeping the temperature uniform on the hot side. The achieved local temperature variations on the cold side has approximately been about $8 \%$ of the temperature difference between the hot and cold sides. The results reveal that the TEG performance shows some variation with the applied variable temperature patterns, which remains, however, rather small for the applied temperature variations. For achieving a more clear answer to the present question, further experiments need to be designed where more substantial temperature variations can be obtained.
\end{abstract}

\section{Nomenclature}

\section{$\vec{E} \quad$ Electric field intensity vector, $\mathrm{V} / \mathrm{m}$ \\ $\vec{i} \quad$ Electric current density vector, $\mathrm{A} / \mathrm{m}$ \\ R Resistance, $\Omega$}

Greek Symbols

$\alpha \quad$ Seebeck coefficient, $\mathrm{V} / \mathrm{K}$

$\sigma \quad$ Electric conductivity, $\mathrm{S} / \mathrm{m}$

$\varphi \quad$ Electric scalar potential, $\mathrm{V}$

\section{Introduction}

One of the key answers to increase the overall efficiency of industrial processes is the waste heat recovery. The energy emission by industrial processes is recognized as low-grade waste heat in many circumstances. This implies that the temperature is often lower than $600{ }^{\circ} \mathrm{C}$. It is nearly impossible to recover this kind of waste heat with conventional heat to electricity conversion systems, like steam or gas turbines.

A conversion process which can deal with low-grade waste heat is the Organic Rankine Cycle (ORC) [1-3], but it is rather complex and involves turbines containing rotary parts [4-6], which, in general, needs a highmaintenance effort.

The thermoelectric generator (TEG) is a waste heat recovery system, which doesn't contain rotary parts and, thus, needs a low maintenance effort. The today's efficiency of around $5 \%$ for the commercial TEGs is a drawback of this technology. Nevertheless, there is an ongoing process in the material research to increase the efficiencies of TEGs and cost optimization of the TEG systems to implement them in various processes optimally with a minimum amortization time. The heat management system around the TEG is a further necessary development, e.g. heat source or cooling, which would decrease the TEG efficiency significantly, if not working in an optimal manner. Even though the TEG cooling system should provide an adequate cooling to achieve a large temperature difference across the TEG and to allow the TEG to work efficiently, the cooling costs should be low to achieve high overall system efficiency.

For the cooling, the major heat transfer mechanism is obviously the convective heat transfer [7], as the encountered temperatures are normally too low for radiation [8] to be important. There are different approaches to convectively cool a TEG. A category is the utilization of the natural convection, where the cooling does not need any power, and, thus, does not influence the system efficiency negatively [9-12].

The alternative approach is the use of forced convection. It is important to minimize the cooling costs for this approach, in order to preserve high system efficiency. For forced convection, there are mostly two working fluids, which are normally considered for practical applications, i.e. air [13] and water [10, 14-17]. Though an amount of electric energy needs to be invested to cool by the forced convection, it is generally observed [14] that a higher overall TEG system efficiency can still be realized in comparison to the natural convection systems.

A constant temperature boundary condition is mostly assumed for the hot and cold side surfaces of the TEG in the practical development of TEG systems, which represents an ideal condition for an optimal behaviour of the TEG. A one-dimensional model for the prediction the performance and efficiency of a TEG can be derived with this assumption, under the consideration of 
temperature independent [18] and temperature dependent [19] material properties.

However, the constant boundary temperature assumption of these simplified, one-dimensional models cannot manage the behaviour of most practical fluid systems that reveal multidimensional, irregular geometries, spatial variations in the flow parameters. Hence, multi-dimensional methods [19-21] are required for being able to perform realistic simulations of practical systems.

There are a few experimentally studies which had the focus on that problem. All related work interconnect several TEG Modules to have scaled more powerful module and the influence of the temperature distribution of over the several modules is investigated.

Montecucco et al. [22] interconnected three TEG of same type, both parallel and serial. Three different Temperature differences $(100 \mathrm{~K}, 150 \mathrm{~K}, 200 \mathrm{~K})$ were applied to the TEG. It turned out that the electrical power decreases about 9.22\%, when the TEG are interconnected in series, compared with to the individual maximum power under this condition when they are not interconnected to each other.

The investigation of Sun et al. [23] extends this approach and take also into account how the reduction of electrical power can be minimized. Due to the usage of additional power inverters with an integrated maximum power point trackers (MPPT) the reduction can be minimized.

The focus of the present paper is the experimental investigation of the effect of TEG performance under the application of a variable temperature pattern on the cold side of a single TEG, to provide a reliable basis for a validated numerical model.

In our previous work, the development of a finite volume method based solver has been presented, relying on the general-purpose, open source $\mathrm{C}++$ toolbox for field operation and manipulation (OpenFOAM) [24].This solver (tegFoam) [25] has also been applied to solve the conjugate heat transfer problem that emerges in the coupled treatment of the TEG with the cooling system [26], where a variable temperature field on the cold side of the TEG emerges.

Those computational results [26] could not be validated against experiments, since relevant measurements could not be found in the open literature, implying the need for such measurements. This need gave the main motivation of the present work. In the present work, measurements are performed by imposing variable temperature distributions on the cold sides of a TEG and performing measurements for different boundary conditions.

\section{Description of the processes in TEG}

Thermoelectric generators (TEG) are solid-state semiconductor apparatus, which, under a prescribed temperature differential, can convert thermal energy, i.e. the heat flowing through, into electrical power. The working principle of a thermoelectric generator is based on the so-called Seebeck effect. The coupled processes of the conductive heat transfer and electric current can mathematically be described by the following coupled non-linear differential field equations with variable material properties [27]

$$
\begin{aligned}
& \nabla \cdot(\mathrm{T} \alpha(\mathrm{T}) \overrightarrow{\mathrm{J}})-\nabla \cdot(\mathrm{k}(\mathrm{T}) \nabla \mathrm{T})=\overrightarrow{\mathrm{J}} \cdot \overrightarrow{\mathrm{E}} \\
& \nabla \cdot(\sigma(\mathrm{T}) \alpha(\mathrm{T}) \nabla \mathrm{T})+\nabla \cdot(\sigma(\mathrm{T}) \nabla \varphi)=0
\end{aligned}
$$

where the electric field intensity vector $(\overrightarrow{\mathrm{E}})$ and electric current density vector $(\overline{\bar{J}})$ are given as

$$
\begin{aligned}
& \overrightarrow{\mathrm{E}}=-\nabla \varphi \\
& \overrightarrow{\mathrm{J}}=\sigma(\mathrm{T})(\overrightarrow{\mathrm{E}}-\alpha \nabla \mathrm{T})
\end{aligned}
$$

The applied temperature difference onto the TEG to generate the heat flow (the temperature difference between the hot and cold sides) is essential for the performance of TEG. In the previous work, we have developed a numerical procedure $[25,26]$ to solve the above equations, which has been validated for conditions, where temperature boundary conditions were spatially homogeneous on each sides. The present work aims to generate data for variable temperature distributions on the cold side of a TEG. The data is planned, to be used in the future, to validate the developed numerical procedure for such conditions.

\section{Experimental}

The influence of the cold side temperature distribution on the performance of a single TEG is measured in a new designed test rig. The test rig is shown in Figures 1 to 3 , the details of which are explained below.

The TEG European Thermodynamics GM200-49-4525 (2) is sandwiched between a hot copper block (1) and the cooling plate (3/4). Four cartridge heaters are mounted in the copper block (1) with an overall maximum power of $800 \mathrm{~W}$. It is assumed that that the temperature in the copper block (1) is constant, due to its thickness and high thermal conductivity. Therefore the temperature is measured at one position with a T-type thermocouple only.

The variation of the cold side is ensured by four different cold plates (4) on top of the TEG. Each of them is cooled with a separate cooler circuit, consisting of a fan (5) EK-Furious Vardar EVO 120, radiator (5) EKCoolStream XE 120 and a pump (6) Magicool DCP450M, so that the temperature of each circuit and cold plate can be controlled by manipulating the speed of the fan and/or pump. The temperature distribution is measured by 8 T-type thermocouples distributed in an additional copper layer (3).

Temperatures are measured in the centre of the cold plates, between these centre point and the border and at the centre of the TEG. The heat transported through each cooling plate is calculated by measuring the inlet and outlet temperature of the cold plates by using T-type 


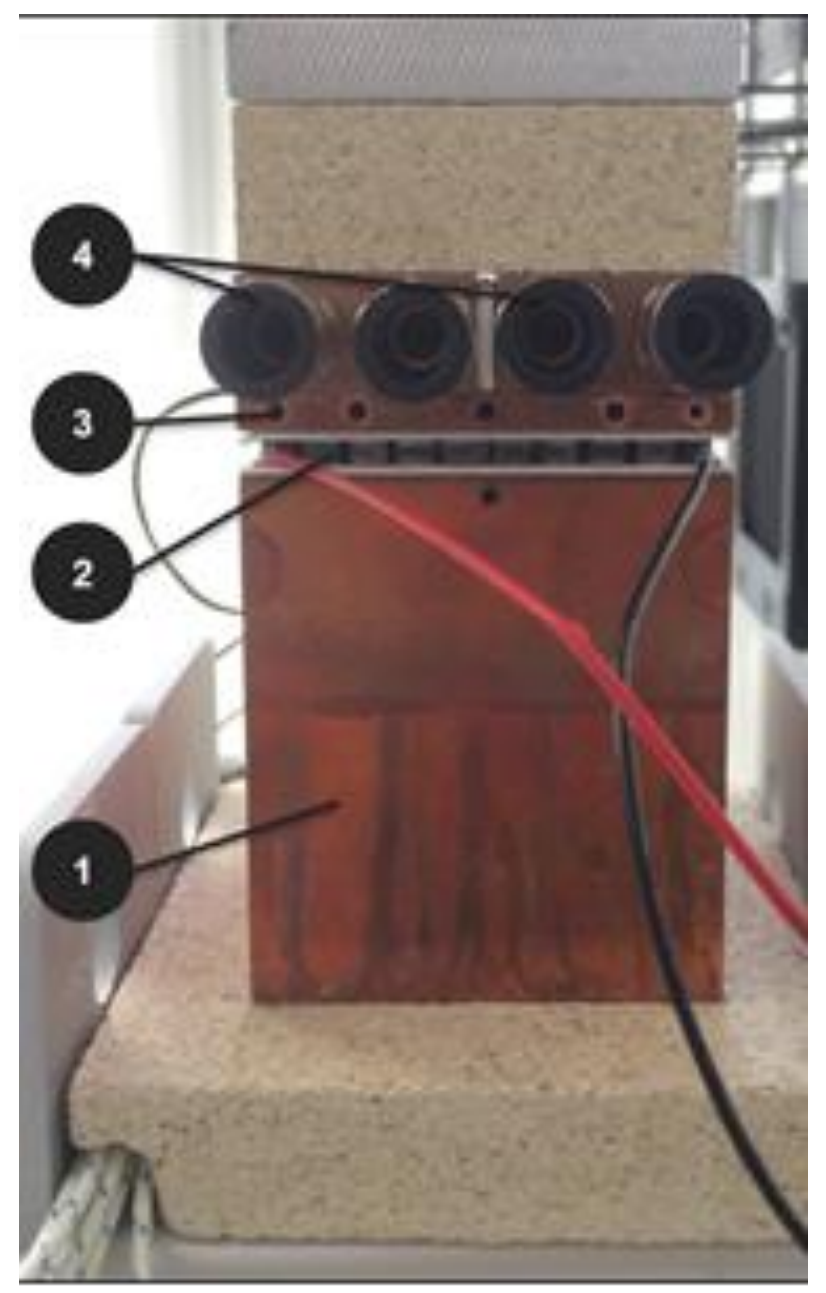

Fig. 1. Test rig: (1) hot copper block (2) TEG (3) measuring plate (4) cold plates

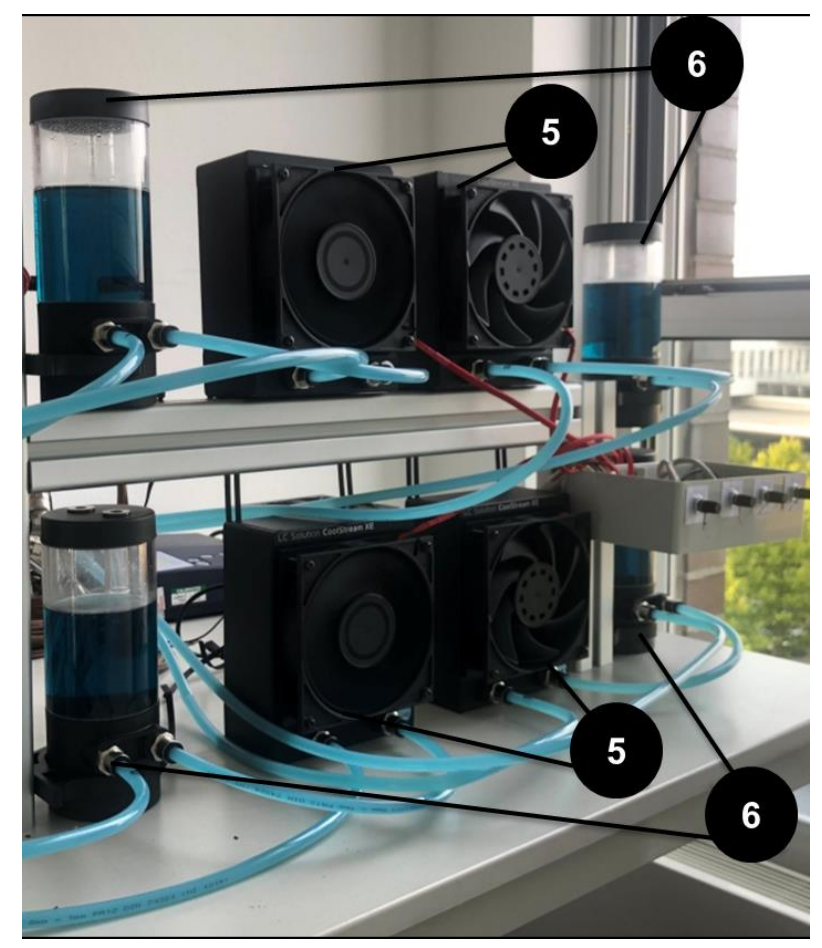

Fig. 2. Test rig: (5) fan and radiator (6) pump

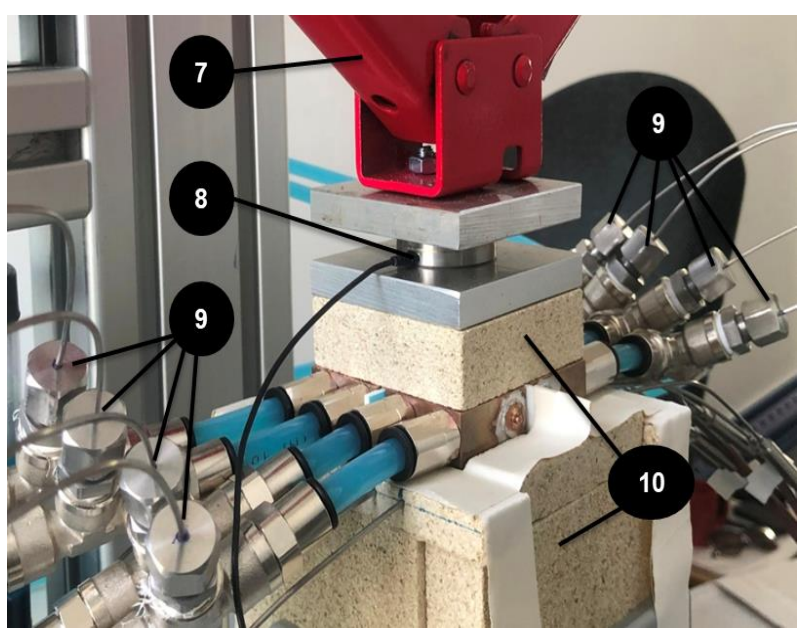

Fig. 3. Test rig: (7) car lifter (8) load cell (9) thermocouples (10) insulation

thermocouples (9) and by measuring the flow rate with a Flexim Fluxus F601. To minimize the thermal contact resistance between hot/cold surface and the surface of the TEG the manufactures of the TEG recommend to apply a specific pressure to the TEG. In this test rig the pressure is applied with a car lifter (7) and simultaneously measured with the load cell (8) Measurement Specialties FC2331-0000-1000-L.

The test rig is insulated with the high temperature and pressure resistant Thermax SF 600 insulation material. Only one layer is used for every side of the hot copper block. This leads to quite high temperatures at the outer surface of the layer and needs to be optimized for future measurements. The thermocouples used have a diameter of $1.5 \mathrm{~mm}$ and are logged with the data logger Grant SQ2020 WiFi.

\section{Results}

During the measurements, the spatial average cold surface temperature is kept at $45^{\circ} \mathrm{C}$ and the hot surface temperature is varied from $75^{\circ} \mathrm{C}$ to $195^{\circ} \mathrm{C}$. Two series are measured, one with a uniform temperature distribution of the cold side and one with a temperature distribution on the cold side, with temperature variation in one spatial direction (each pair of the four cold plates are working under the same condition).

Defining the hot-to-cold temperature difference by $\Delta \mathrm{T}$ (in calculating $\Delta \mathrm{T}$, the spatially averaged temperature is used as the "cold side temperature"), the achieved spatial non-uniformity of the cold side temperature was varying with $\Delta \mathrm{T}$, i.e. increasing nearly proportionally with the latter. On the average it was nearly $8 \%$ of $\Delta \mathrm{T}$, attaining its maximum value, e.g. $12.6^{\circ} \mathrm{C}$, for $\Delta \mathrm{T}=145^{\circ} \mathrm{C}$.

The variations of the measured electrical power produced by TEG for uniform and non-uniform cold side temperature distributions are presented in Figure 4. As seen in the figure, the electrical power by a single TEG increases with the increasing $\Delta \mathrm{T}$, for both cases. It can also be observed that power obtained by the non-uniform distribution is larger that of the uniform distribution, for the medium range of $\Delta \mathrm{T}\left(100^{\circ} \mathrm{C}>\Delta \mathrm{T}>60^{\circ} \mathrm{C}\right)$. 


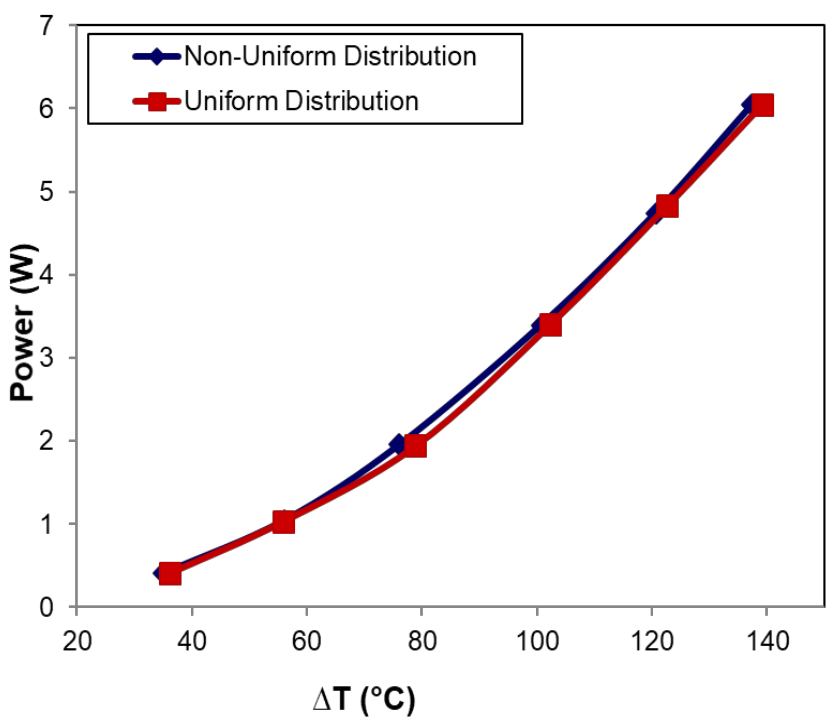

Fig. 4. Electrical power delivered by the TEG using uniform and non-uniform temperature distribution

However, the difference between the both curves is not very substantial, and not necessarily significantly above the range of measurement accuracy. Thus, the present finding may not yet be regarded as conclusive. For achieving a more clear statement, improvements on the experimental set-up need to be done to increase the nonuniformity of the cold-side temperature, and, thereby the measured differences of electrical power between the uniform and non-uniform conditions.

\section{Conclusions}

A sample TEG is analyzed by imposing variable temperature patterns on the cold side, while keeping the temperature uniform on the hot side. The achieved local temperature variations has approximately been about $8 \%$ of the temperature difference between the hot and cold sides. The results show that the TEG performance experiences a variation with temperature non-uniformity. This remains, however, rather small with the applied temperature variations. For achieving a more clear answer, further experiments need to be done with more substantial temperature variations.

\section{References}

1. L. Wang, A.P. Roskilly, R. Wang, "Solar powered cascading cogeneration cycle with ORC and adsorption technology for electricity and refrigeration", Heat Transfer Engineering, 35(1112), pp.1028-1034 (2014)

2. A. Kaya, M. Lazova, Ö. Bagci, S. Lecompte, B. Ameel, M. DePaepe, "Design sensitivity of a platefinned air-cooled condenser for low-temperature organic Rankine cycles", Heat Transfer Engineering, 38(11-12), pp.1018-1033 (2017)

3. Y. Dai, D. Hu, Y. Wu, Y. Gao, Y. Cao, "Comparison of a basic organic Rankine cycle and a parallel double-evaporator organic Rankine-cycle", Heat Transfer Engineering, 38(11-12), pp.990-999 (2017)

4. N. Kamarudin, L.P. Yen, N.W.C. Jusoh, W.S. Ho, J.S. Lim, "Organic Rankine cycle and steam turbine for intermediate temperature waste heat recovery in total site integration", Malaysian J. Fundamental and Applied Sciences, 15(1), pp.125-130 (2019)

5. A.C. Benim, M. Geiger, S. Doehler, M. Schoenenberger, H. Roemer, "Modelling the flow in the exhaust hood of steam turbines under consideration of turbine-exhaust hood interaction", in: Proceed. 1st European. Conf. Turbomachinery Fluid Dynamic and Thermodynamic Aspects: Computational. Methods, Erlangen, Germany, March 1-3, 1995, Book Series: VDI Ber., Vol.1185, pp.343-357 (VDI Verlag, Duesseldorf, 1995)

6. J.G. Andreasen, A. Meroni. F. Haglind, „A comparison of organic and steam rankine cycle power systems for waste heat recovery on large ships“, Energies, 547, 10, doi: 10.3390/en 10040547.

7. W.M. Kays, Convective Heat and Mass Transfer, Tata Mc-Graw-Hill Publ. Comp., New Delhi, 1975)

8. A.C. Benim, "A finite element solution of radiative heat transfer in participating media utilizing the moment method", Computer Methods in Applied Mechanics and Engineering, 67(1), pp.1-14 (1988)

9. C. Wang, C. Hung, W. Chen, "Design of heat sink for improving the performance of thermoelectric generator using two-stage optimization", Energy, 39(1), pp.236-245 (2012)

10. P. Mayer, R. Ram, "Optimization of heat sinklimited thermoelectric generators", Nanoscale and Microscale Thermophysical Engineering, 10(2), pp.143-155 (2006)

11. G. Li, G. Zhang, W. He, J. Ji, S. Lv, X. Chen, H. Chen, "Performance analysis on a solar concentrating thermoelectric generator using the micro-channel heat pipe array", Energy Conversion and Management, 112, pp.191-198 (2016)

12. R.Y. Nuwayhid, A. Shihadeh, N. Ghaddar, "Development and testing of a domestic woodstove thermoelectric generator with natural convection cooling", Energy Conversion and Management, 46(9-10), pp.1631-1643 (2005)

13. M. F. Remeli, A. Date, B. Orr, L. C. Ding, B. Singh, N.D. Affandi, A. Akbarzadeh, "Experimental investigation of combined heat recovery and power generation using a heat pipe assisted thermoelectric generator system", Energy Conversion and Management, 111, pp.147-157 (2016)

14. D.G. Ebling, A. Krumm, B. Pfeiffelmann, J. Gottschald, J. Bruchmann, A.C. Benim, M. Adam, R. Labs, R.R. Herbertz, A. Stunz, "Development of a system for thermoelectric heat recovery from stationary industrial processes", Journal of Electronic Materials, 45(7), pp.3433-3439 (2016)

15. A. Rezania, L.A. Rosendahl, "New configurations of micro plate-fin heat sink to reduce coolant pumping power", Journal of Electronic Materials, 41(6), pp.1298-1304 (2012) 
16. L.C. Ding, N. Meyerheinrich, L. Tan, K. Rahaoui, R. Jain, A. Akbarzadeh, "Thermoelectric Power Generation from Waste Heat of Natural Gas Water Heater", Energy Procedia, 110, pp.32-37 (2017)

17. R. Stobart, M. Wijewardane, Z. Yang, "Comprehensive analysis of thermoelectric generation systems for automotive applications", Applied Thermal Eng., 112, pp.1433-1444 (2017)

18. S.W. Angrist, Direct Energy Conversion, 3rd ed. (Cambridge University Press, Cambridge, 1996)

19. R. Rabari, S. Mahmud, A. Dutta, M. Biglarbegian, "Effect of convection heat transfer on performance of waste water thermoelectric generator", Heat Transfer Engineering, 36(17), pp.1458-1471 (2015)

20. E.E. Antonova, D.C. Looman, "Finite elements for thermoelectric device in ANSYS", Proc. 24th International Thermoelectric Conference, Clemson, SC, USA, pp.200-203 (2005)

21. M. Chen, A. Rosendahl, T.A. Condra, "A threedimensional numerical model of thermoelectric generators in fluid power systems", International Journal of Heat and Mass Transfer, 54(1-3), pp.345355 (2011)

22. A. Montecucco, J. Siviter, A.R. Knox, "The effect of temperature mismatch on thermoelectric generators electrically connected in series and parallel", Applied Energy, 123,pp.47-54 (2014)

23. K. Sun, Z. Qiu, H. Wu, Y. Xing, "Evaluation on High-Efficiency Thermoelectric Generation Systems Based on Differential Power Processing", IEEE Transactions on Industrial Electronics, 65(1), pp.699-708 (2018)

24. The OpenFOAM Foundation. Retrieved October 02, from http://www.openfoam.org/ (2019)

25. B. Pfeiffelmann, A.C. Benim, F. Joos, "A finite volume analysis of thermoelectric generators", Heat Transfer Engineering, 40(17), doi: 10.1080/01457632.2018.1474588 (2019)

26. B. Pfeiffelmann, A.C. Benim, F. Joos, "Numerical analysis of liquid jet impingement cooling of a thermoelectric generator", MATEC Web of Conferences, 240(11-12):01032, doi: 10.1051/matecconf/201824001032 (2018)

27. C. Goupil, (Ed.), Continuum Theory and Modelling of Thermoelectric Elements (Wiley-VCH, Weinheim, Germany, 2016) 